\title{
Synthesis and styrene copolymerization of novel trisubstituted \\ ethylenes: 5. Halogen ring-substituted 2-methoxyethyl \\ phenylcyanoacrylates
}

Grace D. Lee, Mae I. Z. Madrid, Jullisa J. Maya, Andrew S. Nuelle, Nancy Padilla, Gia K. Pappas, Rechal K. Patel, Misael Villegas, Fernanda Aviles, Emily Schroeder, Farheen Shethwala, Jordan Villa, Sara M. Rocus, William S. Schjerven, and Gregory B. Kharas

DePaul University, Chemistry and Biochemistry Department, 1110 West Belden Avenue, Chicago, IL 60614-3214

\begin{abstract}
Novel trisubstituted ethylenes, halogen ring-substituted 2-methoxyethyl phenylcyanoacrylates, $\mathrm{RPhCH}=\mathrm{C}(\mathrm{CN}) \mathrm{CO}_{2} \mathrm{CH}_{2} \mathrm{CH}_{2} \mathrm{OCH}_{3}$ (where $\mathrm{R}$ is 2-bromo, 3-bromo, 4bromo, 2-chloro, 3-chloro, 4-chloro, 2-fluoro, 3-fluoro, 4-fluoro, 2-trifluoromethyl, 3trifluoromethyl, 4-trifluoromethyl) were prepared and copolymerized with styrene. The ethylenes were synthesized by the piperidine catalyzed Knoevenagel condensation of ringsubstituted benzaldehydes and 2-methoxyethyl cyanoacetate, and characterized by CHN analysis, IR, ${ }^{1} \mathrm{H}$ and ${ }^{13} \mathrm{C}$ NMR. All the ethylenes were copolymerized with styrene in
\end{abstract}


solution with radical initiation $(\mathrm{ABCN})$ at $70^{\circ} \mathrm{C}$. The compositions of the copolymers were calculated from nitrogen analysis.

*Address correspondence to: Gregory B. Kharas, Chemistry and Biochemistry Department, DePaul University, Chicago, IL 60614-3214.

\section{Introduction}

Cyanoacrylates is family of vinyl monomers renowned for their high reactivity, instant adhesive properties, and wide-ranging applications [1-3]. Trisubstituted ethylenes (TSE), ring-functionalized phenylcyanoacrylates, $\mathrm{R}^{1} \mathrm{PhCH}=\mathrm{C}(\mathrm{CN}) \mathrm{CO}_{2} \mathrm{R}^{2}(\mathrm{PCA})$ continue to attract attention as compounds with variety of applications [4-13]. Thus, methoxy ringsubstituted methyl phenylcyanoacrylate, MPCA was used in synthesis of pyridotriazines and triazolopyridines [4]. Dimethylamino ring-substituted MPCA was examined among other cyanovinylheteroaromatics in relation to organic nonlinear optics [5]. There are a number of applications of ethyl phenylcyanoacrylate, EPCA and its ring-substituted derivatives, which include studies of catalysis [6] and potential antimicrobial and antioxidant agents [7]. 2,4Dimethoxyphenyl EPCA was used in design, synthesis and study of anticancer activity of novel benzothiazole analogues [8], in synthesis of thiazacridine derivatives as anticancer agents against breast and hematopoietic neoplastic cells [9] and in DABCO-catalyzed Knoevenagel condensation using hydroxy ionic liquid as a promoter [10]. This EPCA was involved in catalysis study of N,N'-dialkylimidazolium dimethyl phosphates [11], in 
synthesis and study of antimicrobial activity of some cyanoacrylates [12], as well as in synthesis of antiproliferative active 2-aminobenzimidazole derivatives [13].

In regards to polymerization reactivity, previous studies showed that PCAs as all TSE monomers containing double bond substituents larger than fluorine have very low reactivity in radical homopolymerization due to polar and steric reasons [14]. Although steric difficulties preclude homopolymerization of such monomers, their copolymerization with a monosubstituted alkenes makes it possible to overcome these steric problems. Thus, copolymerization of electrophylic TSE monomers having double bonds substituted with halo, cyano, and carbonyl groups and electron-rich monosubstituted ethylenes such as styrene, N-vinylcarbazole, and vinyl acetate [15-17] show a tendency toward the formation of alternating copolymers - thus suggesting a way of functionalization of commercial polymers via introduction of isolated monomer units in copolymers.

Earlier we have reported synthesis and styrene copolymerization a number of halogen ring-substituted PCAs, such esters as methyl [18-19], ethyl [20], propyl [21], isopropyl [22], butyl [23], and isobutyl [24]. Our objectives in exploration of novel 2methoxyethyl phenylcyanoacrylates (MEPA) were twofold: (1) to utilize Knoevenagel condensation for synthesis of MEPA compounds with a variety of potentially reactive functional groups and (2) to explore feasibility of radical copolymerization with a commercial monomer styrene.

Thus, in continuation of our investigation of novel TSE compounds we have prepared halogen ring-substituted 2-methoxyethyl phenylcyanoacrylates, $\mathrm{RPhCH}=\mathrm{C}(\mathrm{CN}) \mathrm{CO}_{2} \mathrm{CH}_{2} \mathrm{CH}_{2} \mathrm{OCH}_{3}$, where $\mathrm{R}$ is 2-bromo, 3-bromo, 4-bromo, 2-chloro, 3- 
chloro, 4-chloro, 2-fluoro, 3-fluoro, 4-fluoro, 2-trifluoromethyl, 3-trifluoromethyl, 4trifluoromethyl, and explored the feasibility of their copolymerization with styrene. To the best of our knowledge there have been no reports on either synthesis of these compounds, nor their copolymerization with styrene [25].

\section{Experimental}

\subsection{Materials}

2-Bromo, 3-bromo, 4-bromo, 2-chloro, 3-chloro, 4-chloro, 2-fluoro, 3-fluoro, 4-fluoro, 2trifluoromethyl, 3-trifluoromethyl, 4-trifluoromethyl-substituted benzaldehydes, 2methoxyethyl cyanoacetate ( $\geq 98.0 \%$ ), piperidine (99\%), styrene ( $\geq 99 \%), 1,1^{\prime}-$ azobis(cyclohexanecarbonitrile) (98\%), (ABCN), and toluene (98\%) supplied from SigmaAldrich Co., were used as received.

\subsection{Instrumentation}

Infrared spectra of the MEPA compounds and polymers ( $\mathrm{NaCl}$ plates) were determined with an ABB FTLA 2000 FT-IR spectrometer. The melting points of the MEPA compounds were measured with TA (Thermal Analysis, Inc.) Model Q10 differential scanning calorimeter (DSC). ${ }^{1} \mathrm{H}$ and ${ }^{13} \mathrm{C}$ NMR spectra were obtained on $10-25 \%$ (w/v) MEPA solutions in $\mathrm{CDCl}_{3}$ at ambient temperature using Avance $300 \mathrm{MHz}$ spectrometer. $\mathrm{CHN}$ elemental analyses of MEPA compounds and nitrogen analysis of the copolymers were performed by Midwest Microlab, LLC (IN).

\section{Results and discussion}

\subsection{Synthesis and characterization of 2-methoxyethyl phenylcyanoacrylates}


All MEPA compounds were synthesized by Knoevenagel condensation [26] of appropriate benzaldehydes with 2-methoxyethyl cyanoacetate, catalyzed by base, piperidine (Scheme 1).

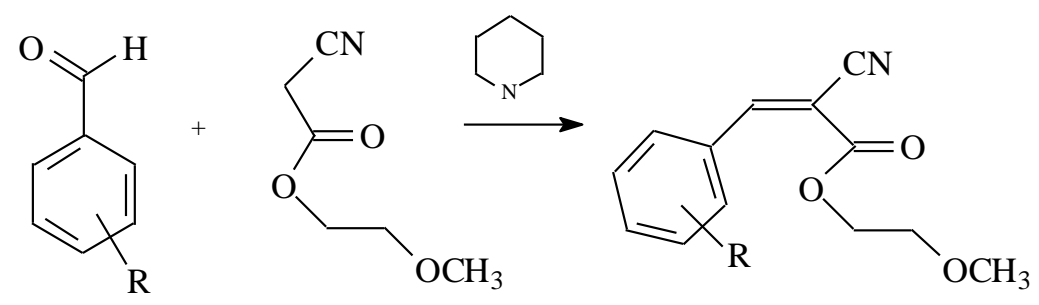

Scheme 1. Synthesis of 2-methoxyethyl phenylcyanoacrylates, where R is 2-bromo, 3bromo, 4-bromo, 2-chloro, 3-chloro, 4-chloro, 2-fluoro, 3-fluoro, 4-fluoro, 2trifluoromethyl, 3-trifluoromethyl, 4-trifluoromethyl.

The preparation procedure was essentially the same for all the MEPA compounds. In a typical synthesis, equimolar amounts of 2-methoxyethyl cyanoacetate and an appropriate benzaldehyde were mixed in equimolar ratio in a $20 \mathrm{~mL}$ vial. A few drops of piperidine were added with stirring. The product of the reaction was isolated by filtration and purified by crystallization from 2-propanol. The condensation reaction proceeded smoothly, yielding products, which were purified by conventional techniques. The compounds were characterized by IR, ${ }^{1} \mathrm{H}$ and ${ }^{13} \mathrm{C}$ NMR spectroscopies. No stereochemical analysis of the novel alkoxy ring-substituted MEPA was performed since no stereoisomers ( $E$ or/and $Z$ ) of known configuration were available.

\subsubsection{2-Methoxyethyl 2-bromophenylcyanoacrylate}


Yield: $82 \%$; mp $56^{\circ} \mathrm{C} ;{ }^{1} \mathrm{H}$ NMR: $\delta 8.3(\mathrm{~s}, 1 \mathrm{H}, \mathrm{CH}=), 8.2-7.4(\mathrm{~m}, 4 \mathrm{H}, \mathrm{Ph}), 4.5(\mathrm{t}, 2 \mathrm{H}$,

$\left.\mathrm{OCOCH}_{2}\right), 3.7\left(\mathrm{t}, 2 \mathrm{H}, \mathrm{OCH}_{2}\right), 3.4\left(\mathrm{~s}, 3 \mathrm{H}, \mathrm{OCH}_{3}\right) ;{ }^{13} \mathrm{C} \mathrm{NMR:} \delta 163(\mathrm{C}=\mathrm{O}), 154(\mathrm{HC}=)$, 136, 132, 129, 128, 127, $115(\mathrm{Ph}), 116(\mathrm{CN}), 106(\mathrm{C}=), 74\left(\mathrm{OCH}_{2}\right), 64\left(\mathrm{OCOCH}_{2}\right), 59$

$\left(\mathrm{OCH}_{3}\right)$; IR: $\left(\mathrm{cm}^{-1}\right) 2992$ (m, C-H), 2228 (m, CN), 1734 (s, C=O), 1585 (s, C=C), 1263

(s, C-O-CH 3 ), 708 (s, C-H out of plane). Anal. calcd. for $\mathrm{C}_{13} \mathrm{H}_{12} \mathrm{BrNO}_{3}$ : C, 50.34; $\mathrm{H}$,

$3.90 ;$ N, 4.52; Found: C, 48.14; H, 3.86; N, 4.54.

\subsubsection{2-Methoxyethyl 3-bromophenylcyanoacrylate}

Yield: 95\%; mp 56.2 ${ }^{\circ} \mathrm{C} ;{ }^{1} \mathrm{H}$ NMR: $\delta 8.2(\mathrm{~s}, 1 \mathrm{H}, \mathrm{CH}=), 8.1-7.3(\mathrm{~m}, 4 \mathrm{H}, \mathrm{Ph}), 4.5(\mathrm{t}, 2 \mathrm{H}$, $\left.\mathrm{OCOCH}_{2}\right), 3.7\left(\mathrm{t}, 2 \mathrm{H}, \mathrm{OCH}_{2}\right), 3.4\left(\mathrm{~s}, 3 \mathrm{H}, \mathrm{OCH}_{3}\right) ;{ }^{13} \mathrm{C} \mathrm{NMR}: \delta 162(\mathrm{C}=\mathrm{O}), 153(\mathrm{HC}=)$, 132, 131, $130(\mathrm{Ph}), 116(\mathrm{CN}), 100(\mathrm{C}=), 64\left(\mathrm{OCOCH}_{2}\right), 59\left(\mathrm{OCH}_{3}\right)$; IR: $\left(\mathrm{cm}^{-1}\right) 2982(\mathrm{~m}$, C-H), 2224 (m, CN), 1747 (s, C=O), 1609 (s, C=C), 1257 (s, C-O-CH 3 ), 781, 677 (s, C-H out of plane). Anal. calcd. for $\mathrm{C}_{13} \mathrm{H}_{12} \mathrm{BrNO}_{3}$ : C, 50.34; H, 3.90; N, 4.52; Found: C, 48.03; $\mathrm{H}, 3.63 ; \mathrm{N}, 4.57$.

\subsubsection{2-Methoxyethyl 4-bromophenylcyanoacrylate}

Yield $73 \%$; mp $42.4^{\circ} \mathrm{C} ;{ }^{1} \mathrm{H}$ NMR $\delta 8.2(\mathrm{~s}, 1 \mathrm{H}, \mathrm{CH}=), 7.9-6.9(\mathrm{~m}, 4 \mathrm{H}, \mathrm{Ph}), 4.8(\mathrm{t}, 2 \mathrm{H}$,

$\left.\mathrm{OCOCH}_{2}\right), 3.7\left(\mathrm{t}, 2 \mathrm{H}, \mathrm{OCH}_{2}\right), 3.4\left(\mathrm{~s}, 3 \mathrm{H}, \mathrm{OCH}_{3}\right) ;{ }^{13} \mathrm{C} \mathrm{NMR}: \delta 162(\mathrm{C}=\mathrm{O}), 154(\mathrm{HC}=)$, 132, 131, $130(\mathrm{Ph}), 115(\mathrm{CN}), 103(\mathrm{C}=), 70\left(\mathrm{OCH}_{2}\right), 66\left(\mathrm{OCOCH}_{2}\right), 59\left(\mathrm{OCH}_{3}\right) ; \mathrm{IR}:\left(\mathrm{cm}^{-}\right.$ 1) $2928(\mathrm{~m}, \mathrm{C}-\mathrm{H}), 2220(\mathrm{~m}, \mathrm{CN}), 1732(\mathrm{~s}, \mathrm{C}=\mathrm{O}), 1603$ (s, C=C), 1257 (s, C-O-CH3), 791 (s, C-H out of plane). Anal. calcd. for $\mathrm{C}_{13} \mathrm{H}_{12} \mathrm{BrNO}_{3}$ : C, 50.34; H, 3.90; N, 4.52; Found: C, 48.09; H, 3.77; N, 4.19.

\subsubsection{2-Methoxyethyl 2-chlorophenylcyanoacrylate}


Yield $82 \%$; ${ }^{1} \mathrm{H}$ NMR $\delta 8.2(\mathrm{~s}, 1 \mathrm{H}, \mathrm{CH}=), 7.9-7.3(\mathrm{~m}, 4 \mathrm{H}, \mathrm{Ph}), 4.3\left(\mathrm{t}, 2 \mathrm{H}, \mathrm{OCOCH}_{2}\right), 3.7$

$\left(\mathrm{t}, 2 \mathrm{H}, \mathrm{OCH}_{2}\right), 3.4\left(\mathrm{~s}, 3 \mathrm{H}, \mathrm{CH}_{3} \mathrm{O}\right) ;{ }^{13} \mathrm{C} \mathrm{NMR} \delta 164(\mathrm{C}=\mathrm{O}), 155(\mathrm{HC}=), 134,133,130$, $129(\mathrm{Ph}), 115(\mathrm{CN}), 106(\mathrm{C}=), 70\left(\mathrm{OCH}_{2}\right), 66\left(\mathrm{OCOCH}_{2}\right), 59\left(\mathrm{OCH}_{3}\right) ; \mathrm{IR}\left(\mathrm{cm}^{-1}\right): 2884$ (m, C-H), 2226 (m, CN), 1732 (s, C=O), 1609 (s, C=C), 1203 (s, C-O-CH3), 862, 750 (s, C-H out of plane). Anal. Calcd. for $\mathrm{C}_{13} \mathrm{H}_{12} \mathrm{ClNO}_{3}$ : C, 58.77; H, 4.55; N, 5.27; Found: C, 54.77; H, 4.64; N, 5.17.

\subsubsection{2-Methoxyethyl 3-chlorophenyl)phenylcyanoacrylate}

Yield $72 \%$; mp $58.1^{\circ} \mathrm{C} ;{ }^{1} \mathrm{H}$ NMR $\delta 8.2(\mathrm{~s}, 1 \mathrm{H}, \mathrm{CH}=), 7.9-7.3(\mathrm{~m}, 4 \mathrm{H}, \mathrm{Ph}), 4.8(\mathrm{t}, 2 \mathrm{H}$, $\left.\mathrm{OCOCH}_{2}\right), 3.7\left(\mathrm{t}, 2 \mathrm{H}, \mathrm{OCH}_{2}\right), 3.4\left(\mathrm{~s}, 3 \mathrm{H}, \mathrm{CH}_{3} \mathrm{O}\right) ;{ }^{13} \mathrm{C} \mathrm{NMR} \delta 162(\mathrm{C}=\mathrm{O}), 153(\mathrm{HC}=)$, 137, 135, 134, 130, $129(\mathrm{Ph}), 115(\mathrm{CN}), 105(\mathrm{C}=), 70\left(\mathrm{OCH}_{2}\right), 66\left(\mathrm{OCOCH}_{2}\right), 59$ $\left(\mathrm{OCH}_{3}\right) ; \mathrm{IR}\left(\mathrm{cm}^{-1}\right): 2945(\mathrm{~m}, \mathrm{C}-\mathrm{H}), 2226(\mathrm{~m}, \mathrm{CN}), 1717$ (s, C=O), 1609 (s, C=C), 1271 (s, C-O-CH 3 ), 851, 787 (s, C-H out of plane). Anal. Calcd. for $\mathrm{C}_{13} \mathrm{H}_{12} \mathrm{ClNO}_{3}: \mathrm{C}, 58.77 ; \mathrm{H}$, 4.55; N, 5.27; Found: C, 55.63; H, 4.51; N, 5.40.

\subsubsection{2-Methoxyethyl 4-chlorophenoxy)phenylcyanoacrylate}

Yield $87 \%$; mp $86.9^{\circ} \mathrm{C} ;{ }^{1} \mathrm{H}$ NMR $\delta 8.2(\mathrm{~s}, 1 \mathrm{H}, \mathrm{CH}=), 8.1-7.3(\mathrm{~m}, 4 \mathrm{H}, \mathrm{Ph}), 4.8(\mathrm{t}, 2 \mathrm{H}$, $\left.\mathrm{OCOCH}_{2}\right), 3.7\left(\mathrm{t}, 2 \mathrm{H}, \mathrm{OCH}_{2}\right), 3.4\left(\mathrm{~s}, 3 \mathrm{H}, \mathrm{CH}_{3} \mathrm{O}\right) ;{ }^{13} \mathrm{C} \mathrm{NMR} \delta 162(\mathrm{C}=\mathrm{O}), 154(\mathrm{HC}=)$, 140, 132, 131, 130, $129(\mathrm{Ph}), 115(\mathrm{CN}), 103(\mathrm{C}=), 70\left(\mathrm{OCH}_{2}\right), 66\left(\mathrm{OCOCH}_{2}\right), 59$ $\left(\mathrm{OCH}_{3}\right)$; IR (cm $\left.{ }^{-1}\right): 2899$ (m, C-H), 2222 (m, CN), 1724 (s, C=O), 1612 (s, C=C), 1290 (s, C-O-CH 3 ), 829, 760 (s, C-H out of plane). Anal. Calcd. for $\mathrm{C}_{13} \mathrm{H}_{12} \mathrm{ClNO}_{3}: \mathrm{C}, 58.77 ; \mathrm{H}$, 4.55; N, 5.27; Found: C, 56.06; H, 4.63; N, 5.60.

\subsubsection{2-Methoxyethyl 2-fluorophenylcyanoacrylate}


Yield 84; ${ }^{1} \mathrm{H}$ NMR $\delta 8.6(\mathrm{~s}, 1 \mathrm{H}, \mathrm{CH}=), 8.4-7.0(\mathrm{~m}, 4 \mathrm{H}, \mathrm{Ph}), 4.5\left(\mathrm{t}, 2 \mathrm{H}, \mathrm{OCOCH}_{2}\right), 3.7(\mathrm{t}$, $\left.2 \mathrm{H}, \mathrm{OCH}_{2}\right), 3.4\left(\mathrm{~s}, 3 \mathrm{H}, \mathrm{CH}_{3} \mathrm{O}\right) ;{ }^{13} \mathrm{C} \mathrm{NMR} \delta 163(\mathrm{C}=\mathrm{O}), 154(\mathrm{HC}=), 146,135,129,125$, $120(\mathrm{Ph}), 117(\mathrm{CN}), 105(\mathrm{C}=), 70\left(\mathrm{OCH}_{2}\right), 65\left(\mathrm{OCOCH}_{2}\right), 59\left(\mathrm{OCH}_{3}\right) ; \mathrm{IR}\left(\mathrm{cm}^{-1}\right): 2934$ (m, C-H), 2228 (m, CN), 1742 (s, C=O), 1610 (s, C=C), 1236 (s, C-O-CH3), 804 (s, C-H out of plane). Anal. Calcd. for $\mathrm{C}_{13} \mathrm{H}_{12} \mathrm{FNO}_{3}$ : C, 62.65; H, 4.85; N, 5.62; Found: C, 63.42; H, 4.61; N, 5.49.

\subsubsection{2-Methoxyethyl 3-fluorophenylcyanoacrylates}

Yield 78\%; ${ }^{1} \mathrm{H}$ NMR $\delta 8.2(\mathrm{~s}, 1 \mathrm{H}, \mathrm{CH}=), 7.8-7.0(\mathrm{~m}, 4 \mathrm{H}, \mathrm{Ph}), 4.5\left(\mathrm{t}, 2 \mathrm{H}, \mathrm{OCOCH}_{2}\right), 3.7$

$\left(\mathrm{t}, 2 \mathrm{H}, \mathrm{OCH}_{2}\right), 3.4\left(\mathrm{~s}, 3 \mathrm{H}, \mathrm{CH}_{3} \mathrm{O}\right) ;{ }^{13} \mathrm{C} \mathrm{NMR} \delta 163(\mathrm{C}=\mathrm{O}), 153(\mathrm{HC}=), 133,132,127$, 122, 120, $117(\mathrm{Ph}), 116(\mathrm{CN}), 104(\mathrm{C}=), 70\left(\mathrm{OCH}_{2}\right), 65\left(\mathrm{OCOCH}_{2}\right), 59\left(\mathrm{OCH}_{3}\right)$; IR $\left(\mathrm{cm}^{-}\right.$

1): 2935 (m, C-H), 2224 (m, CN), 1734 (s, C=O), 1614 (s, C=C), 1227 (s, C-O-CH3), 872, 762 (s, C-H out of plane). Anal. Calcd. for $\mathrm{C}_{13} \mathrm{H}_{12} \mathrm{FNO}_{3}$ : C, 62.65; H, 4.85; N, 5.62;

Found: C, 58.15; H, 4.75; N, 5.55.

\subsubsection{2-Methoxyethyl 4-fluorophenylcyanoacrylate}

Yield 77\%; ${ }^{1} \mathrm{H}$ NMR $\delta 8.2(\mathrm{~s}, 1 \mathrm{H}, \mathrm{CH}=), 8.1-7.1(\mathrm{~m}, 4 \mathrm{H}, \mathrm{Ph}), 4.5\left(\mathrm{t}, 2 \mathrm{H}, \mathrm{OCOCH}_{2}\right), 3.7$

$\left(\mathrm{t}, 2 \mathrm{H}, \mathrm{OCH}_{2}\right), 3.5\left(\mathrm{~s}, 3 \mathrm{H}, \mathrm{CH}_{3} \mathrm{O}\right) ;{ }^{13} \mathrm{C} \mathrm{NMR} \delta 164(\mathrm{C}=\mathrm{O}), 154(\mathrm{HC}=), 133,129,116$ (Ph), $115(\mathrm{CN}), 102(\mathrm{C}=), 70\left(\mathrm{OCH}_{2}\right), 65\left(\mathrm{OCOCH}_{2}\right), 62\left(\mathrm{OCH}_{3}\right) ; \mathrm{IR}\left(\mathrm{cm}^{-1}\right): 2966(\mathrm{~m}$, C-H), 2224 (m, CN), 1720 (s, C=O), 1597 (s, C=C), 1240 (s, C-O-CH 3 ), 841 (s, C-H out of plane). Anal. Calcd. for $\mathrm{C}_{13} \mathrm{H}_{12} \mathrm{FNO}_{3}: \mathrm{C}, 62.65 ; \mathrm{H}, 4.85 ; \mathrm{N}, 5.62$; Found: $\mathrm{C}, 60.33 ; \mathrm{H}$, 4.96; N, 5.85.

\subsubsection{2-Methoxyethyl 2-trifluoromethylphenylcyanoacrylates}


Yield 91\%; ${ }^{1} \mathrm{H}$ NMR $\delta 8.1(\mathrm{~s}, 1 \mathrm{H}, \mathrm{CH}=), 7.9-7.6(\mathrm{~m}, 4 \mathrm{H}, \mathrm{Ph}), 4.3\left(\mathrm{t}, 2 \mathrm{H}, \mathrm{OCOCH}_{2}\right), 3.6$

$\left(\mathrm{t}, 2 \mathrm{H}, \mathrm{OCH}_{2}\right), 3.4\left(\mathrm{~s}, 3 \mathrm{H}, \mathrm{CH}_{3} \mathrm{O}\right) ;{ }^{13} \mathrm{C} \mathrm{NMR} \delta 164(\mathrm{C}=\mathrm{O}), 152(\mathrm{HC}=), 133,132,131,126$

$(\mathrm{Ph}), 114(\mathrm{CN}), 125\left(\mathrm{CF}_{3}\right), 120(\mathrm{C}=), 70\left(\mathrm{OCH}_{2}\right), 64\left(\mathrm{OCOCH}_{2}\right), 59\left(\mathrm{OCH}_{3}\right) ; \mathrm{IR}\left(\mathrm{cm}^{-1}\right)$ :

2935 (m, C-H), 2231 (m, CN), 1749 (s, C=O), 1601 (s, C=C), 1296 (s, C-O-CH ), 819,

771 (s, C-H out of plane). Anal. Calcd. for $\mathrm{C}_{14} \mathrm{H}_{12} \mathrm{~F}_{3} \mathrm{NO}_{3}$ : C, 56.19; H, 4.04; N, 4.68;

Found: C, 52.98; H, 4.42; N, 4.44.

\subsubsection{2-Methoxyethyl 3-trifluoromethylphenylcyanoacrylates}

Yield $81 \%$; ${ }^{1} \mathrm{H}$ NMR $\delta 8.3(\mathrm{~s}, 1 \mathrm{H}, \mathrm{CH}=), 8.2-7.5(\mathrm{~m}, 4 \mathrm{H}, \mathrm{Ph}), 4.5\left(\mathrm{t}, 2 \mathrm{H}, \mathrm{OCOCH}_{2}\right), 3.7$

(t, 2H, $\left.\mathrm{OCH}_{2}\right), 3.4\left(\mathrm{~s}, 3 \mathrm{H}, \mathrm{CH}_{3} \mathrm{O}\right) ;{ }^{13} \mathrm{C}$ NMR $\delta 163(\mathrm{C}=\mathrm{O}), 153(\mathrm{HC}=), 137,133,132,124$

$(\mathrm{Ph}), 125\left(\mathrm{CF}_{3}\right), 116(\mathrm{CN}), 105(\mathrm{C}=), 69\left(\mathrm{OCH}_{2}\right), 64\left(\mathrm{OCOCH}_{2}\right), 59\left(\mathrm{OCH}_{3}\right) ; \mathrm{IR}\left(\mathrm{cm}^{-1}\right)$ :

2914 (m, C-H), 2235 (m, CN), 1718 (s, C=O), 1609 (s, C=C), 1227 (s, C-O-CH ), 866,

806, 762 (s, C-H out of plane). Anal. Calcd. for $\mathrm{C}_{14} \mathrm{H}_{12} \mathrm{~F}_{3} \mathrm{NO}_{3}$ : C, 56.19; H, 4.04; N, 4.68;

Found: C, 52.98; H, 4.42; N, 4.44.

\subsubsection{2-Methoxyethyl 4-trifluoromethylphenylcyanoacrylates}

Yield 74\%; mp $78.6^{\circ} \mathrm{C}$; ${ }^{1} \mathrm{H}$ NMR $\delta 8.3(\mathrm{~s}, 1 \mathrm{H}, \mathrm{CH}=), 8.2-7.7(\mathrm{~m}, 4 \mathrm{H}, \mathrm{Ph}), 4.5(\mathrm{t}, 2 \mathrm{H}$,

$\left.\mathrm{OCOCH}_{2}\right), 3.7\left(\mathrm{t}, 2 \mathrm{H}, \mathrm{OCH}_{2}\right), 3.5\left(\mathrm{~s}, 3 \mathrm{H}, \mathrm{CH}_{3} \mathrm{O}\right) ;{ }^{13} \mathrm{C} \mathrm{NMR} \delta 162(\mathrm{C}=\mathrm{O}), 153(\mathrm{HC}=)$,

134, 133, $131(\mathrm{Ph}), 126\left(\mathrm{CF}_{3}\right), 115(\mathrm{CN}), 106(\mathrm{C}=), 70\left(\mathrm{OCH}_{2}\right), 66\left(\mathrm{OCOCH}_{2}\right), 59$

$\left(\mathrm{OCH}_{3}\right)$; IR ( $\left.\mathrm{cm}^{-1}\right): 2955$ (m, C-H), $2228(\mathrm{~m}, \mathrm{CN}), 1726$ (s, C=O), 1616 (s, C=C), 1121

(s, C-O-CH3), 849, 764, 608 (s, C-H out of plane). Anal. Calcd. for $\mathrm{C}_{14} \mathrm{H}_{12} \mathrm{~F}_{3} \mathrm{NO}_{3}$ : C,

56.19; H, 4.04; N, 4.68; Found: C, 51.29; H, 3.86; N, 4.84. 


\subsection{Homopolymerization}

An attempted homopolymerization of the MEPA compounds in the presence of $\mathrm{ABCN}$ did not produce any polymer as indicated by the lack of a precipitate in methanol. The inability of the monomers to polymerize is associated with steric difficulties encountered in homopolymerization of 1,1- and 1,2-disubstituted ethylenes [14]. Homopolymerization of styrene (ST) under conditions identical to those in copolymerization experiments yielded $18.3 \%$ of polystyrene, when polymerized for $30 \mathrm{~min}$.

\subsection{Synthesis and characterization of styrene-MEPA copolymers}

Copolymers of the ST and the MEPA compounds, P(ST-co-MEPA) were prepared in 25-

$\mathrm{mL}$ glass screw cap vials at ST/MEPA $=3(\mathrm{~mol})$ the monomer feed using $0.12 \mathrm{~mol} / \mathrm{L}$ of $\mathrm{ABCN}$ at an overall monomer concentration $2.44 \mathrm{~mol} / \mathrm{L}$ in $10 \mathrm{~mL}$ of toluene. The copolymerization was conducted at $70^{\circ} \mathrm{C}$. After a predetermined time, the mixture was cooled to room temperature, and precipitated dropwise in methanol. The composition of the copolymers was determined based on the nitrogen content (cyano group in MEPA monomers). The novel synthesized MEPA compounds copolymerized readily with ST under free-radical conditions (Scheme 2) forming white flaky precipitates when their solutions were poured into methanol. The conversion of the copolymers was kept between 10 and $20 \%$ to minimize compositional drift (Table 1). 


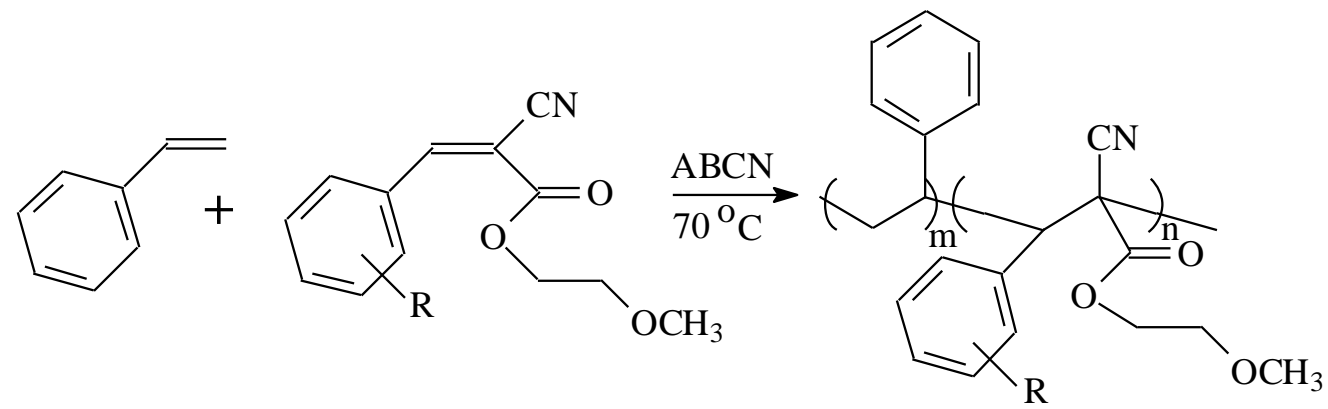

Scheme 2. Copolymerization of ST and halogen ring-substituted 2-methoxyethyl phenylcyanoacrylates, $\mathrm{RPhCH}=\mathrm{C}(\mathrm{CN}) \mathrm{CO}_{2} \mathrm{CH}_{2} \mathrm{CH}_{2} \mathrm{OCH}_{3}$, where $\mathrm{R}$ is 2-bromo, 3-bromo, 4-bromo, 2-chloro, 3-chloro, 4-chloro, 2-fluoro, 3-fluoro, 4-fluoro, 2-trifluoromethyl, 3trifluoromethyl, 4-trifluoromethyl.

Table 1. Copolymerization of Styrene and 2-Methoxyethyl phenylcyanoacrylates.

\begin{tabular}{|l|c|c|c|c|}
\hline \multicolumn{1}{|c|}{$\mathrm{R}$} & $\begin{array}{c}\text { Yield }^{\mathrm{a}} \\
(\mathrm{wt} \%)\end{array}$ & $\begin{array}{c}\mathrm{N} \\
(\mathrm{wt} \%)\end{array}$ & $\begin{array}{c}\text { ST in } \\
\text { copol. } \\
(\mathrm{mol} \%)\end{array}$ & $\begin{array}{c}\text { MEPA in } \\
\text { copol. } \\
(\mathrm{mol} \%)\end{array}$ \\
\hline 2-Bromo & 11.2 & 2.32 & 73.8 & 26.2 \\
\hline 3-Bromo & 14.3 & 2.51 & 70.4 & 29.6 \\
\hline 4-Bromo & 12.3 & 2.36 & 73.1 & 26.9 \\
\hline 2-Chloro & 16.7 & 2.56 & 73.0 & 27.0 \\
\hline 3-Chloro & 15.2 & 2.64 & 71.8 & 28.2 \\
\hline 4-Chloro & 12.3 & 2.55 & 73.2 & 26.8 \\
\hline 2-Fluoro & 14.4 & 2.63 & 73.1 & 26.9 \\
\hline 3-Fluoro & 12.9 & 2.64 & 73.0 & 27.0 \\
\hline 4-Fluoro & 14.5 & 2.58 & 73.8 & 26.2 \\
\hline 2-Trifluoromethyl & 12.6 & 1.75 & 82.8 & 17.2 \\
\hline 3-Trifluoromethyl & 13.5 & 2.12 & 77.7 & 22.3 \\
\hline 4-Trifluoromethyl & 14.4 & 2.25 & 75.7 & 24.3 \\
\hline
\end{tabular}

Nitrogen elemental analysis showed that between 17.2 and $29.6 \mathrm{~mol} \%$ of MEPA is present in the copolymers prepared at ST/MEPA $=3(\mathrm{~mol})$, which is indicative of 
relatively high reactivity of the MEPA monomers towards ST radical which is typical of phenoxy ring-substituted phenylcyanoacrylates [18-24]. Since MEPA monomers do not homopolymerize, the most likely structure of the copolymers would be isolated MEPA monomer units alternating with short ST sequences (Scheme 2).

The copolymers prepared in the present work are all soluble in ethyl acetate, THF, DMF and $\mathrm{CHCl}_{3}$ and insoluble in methanol, ethyl ether, and petroleum ether.

\section{Conclusions}

Novel trisubstituted ethylenes, halogen ring-substituted 2-methoxyethyl phenylcyanoacrylates, $\mathrm{RPhCH}=\mathrm{C}(\mathrm{CN}) \mathrm{CO}_{2} \mathrm{CH}_{2} \mathrm{CH}_{2} \mathrm{OCH}_{3}$ (where $\mathrm{R}$ is 2-bromo, 3-bromo, 4-bromo, 2-chloro, 3-chloro, 4-chloro, 2-fluoro, 3-fluoro, 4-fluoro, 2-trifluoromethyl, 3trifluoromethyl, 4-trifluoromethyl) were prepared and copolymerized with styrene.

\section{Acknowledgments}

The authors are grateful to acknowledge that the project was partly supported by Chicago Society of Coating Technology (CSCT).

\section{References}

[1] Klemarczyk, P.; Guthrie, J. Advances in anaerobic and cyanoacrylate adhesives. In Advances in Structural Adhesive Bonding, 1st ed.; Dillard, D., Ed.; Woodhead Publishing Limited: Cambridge, UK, 2010; pp. 96-131, ISBN 978-1-84569-435-7. 
[2] Shantha, K.L.; Thennarasu, S.; Krishnamurti, N. Developments and applications of cyanoacrylate adhesives. J. Adhes. Sci. Technol. 1989, 3, 237-260.

[3] J.M. Korde and B. Kandasubramanian, Biocompatible alkyl cyanoacrylates and their derivatives as bio-adhesives, Biomaterials Science, 10.1039/C8BM00312B, 6, 7, (1691-1711), (2018).

[4] Zaki MEA, Fathalla OA, Swelam SA, Aly HF (2004) Synthesis of pyrido[2,1c] $[1,2,4]$ triazine, 1,2,4-triazolo[4,3-a]pyridine and 2-(pyrazolyl)nicotinonitrile and their effect on Biomphalaria alexandrina snail enzymes. Acta Poloniae Pharmaceutica 61: $55-64$.

[5] Matsuoka M, Takao M, Kitao T, Fujiwara T, Nakatsu K (1990) Cyanovinylheteroaromatics for Organic Nonlinear Optics. Molecular Crystals and Liquid Crystals, 182A: 71-79.

[6] Burate PA, Javle BR, Desale PH, Kinage AK (2019) Amino acid amide based ionic liquid as an efficient organo-catalyst for solvent-free Knoevenagel condensation at room temperature. Catalysis Letters 149(9): 2368-2375.

[7] Medyouni R, Hamdi N, Ben Said R, Al-Ayed AS, Zagrouba F (2013) Clean procedure and DFT study for the synthesis of 2-amino-3-ethoxycarbonyl-4-(aryl)-4Hpyrano-[3,2-c]-chromene-5-ones derivatives: A novel class of potential antimicrobial and antioxidant agents. Journal of Chemistry 2013: 1-4. 
[8] Hassan AY, Sarg MT, Hussein EM (2019) Design, synthesis and anticancer activity of novel benzothiazole analogues. Journal of Heterocyclic Chemistry 56(4): 14371457.

[9] Moacyr JB, De Melo R, Wanessa LB, De Sena, RO, De Moura, Iris TT et al. (2017). Synthesis and anticancer evaluation of thiazacridine derivatives reveals new selective molecules to hematopoietic neoplastic cells. Combinatorial Chemistry \& High Throughput Screening 20(8): 713.

[10] Meng D, Qiao Y, Wang X, Wen W, Zhao S (2018) DABCO-catalyzed Knoevenagel condensation of aldehydes with ethyl cyanoacetate using hydroxy ionic liquid as a promoter. RSC Advances 8(53): 30180-30185.

[11] Brica S, Freimane L, Kulikovska L, Zicmanis A (2017) N,N'-dialkylimidazolium dimethyl phosphates - promising media and catalysts at the same time for condensation reactions. Chemical Science International Journal 19(4): 1-9.

[12] Bhuiyan M, Mosharef H, Rahman KM, Alam MA, Mahmud M (2013) Microwave assisted Knoevenagel condensation: synthesis and antimicrobial activities of some a-cyanoacrylates. Pakistan Journal of Scientific and Industrial Research, Series A: Physical Sciences 56(3): 131-137.

[13] Nowicka A, Liszkiewicz H, Nawrocka WP, Wietrzyk J, Kempinska K, Drys A (2014) Synthesis and antiproliferative activity in vitro of new 2-aminobenzimidazole derivatives. Reaction of 2-arylideneaminobenzimidazole with selected nitriles 
containing active methylene group. Central European Journal of Chemistry 12(10): 1047-1055.

[14] Odian, G. Principles of Polymerization, 4th Ed., Wiley-Interscience: New York, 2004.

[15] Hall, H. K., Jr.; Padias, A. B. J. Polym. Sci.Part A: Polym. Chem. 2004, 42, 28452858.

[16] Hall, H. K. Jr.; Ykman, P. Macromolecules. 1977, 10, 464.

[17] Kharas, G. B. Trisubstituted Ethylene Copolymers. In Polymeric Materials Encyclopedia, Salamone, J.C., (Ed.) CRC Press: Boca Raton, FL, 11, 8405, 1996.

[18] Novel Copolymers of Trisubstituted Ethylenes with Styrene: 2. Halogen Phenyl Substituted Methyl 2-cyano-3-phenyl-2-propenoates. G.B. Kharas, Wheeler, T.S., Eaker, J.M., Armatys, S.A., Fehringer, J., Gehant, R., Glaser, E., Johnson, K., Moy, P., Quinting, G.R., Macromolecular Reports, A32, $405-414$ (1995).

[19] Synthesis and Copolymerization of Halogen Phenyl-substituted Methyl 2-Cyano-3phenyl-2-propenoates with Styrene. K. Kim, C.A. Butler, M.R. Cisneros, S.L. Ryan, M.A. Schwartz, N.A. Lindquist, G.B. Kharas, J.V. DeFrancesco. Polymer Bull., 40, 361-365 (1998).

[20] Novel Copolymers of Styrene. 3. Halogen Ring-substituted Ethyl 2-Cyano-3phenyl-2-propenoates. G.B. Kharas, E.S. Molina, K.E. Abma, R.A. Ali, K.D. Bairaktaris, F.P. Biasiello, E.A. Cygan, J. Gibson, S. Haq, D.E. Hoag, K. Johnson, S.I. Jordan, M.B. Mathews, C.A. Shadduck, N. Tuzik. J.Macromol. Sci. A50 (2) 139-143 (2013). 
[21] Novel Copolymers of Styrene. 4. Halogen Ring-Substituted Propyl 2-Cyano-3-

Phenyl-2-Propenoates. G.B. Kharas, V.A. Sloan-Lyon, J.D. Grannum, M.B. Gudger, M.L.

Isai, L.A. Kopczynski, C.N. Lynch, H. M. Meyer, B.A. Roman, S.R. Schaefer, and L.F.

Welk. J. Macromol. Sci. A53(11) 659-663 (2016).

[22] Synthesis and styrene copolymerization of halogen ring-substituted isopropyl cyanophenyl propenoates. B.Y. Killam, T.S. Bullock, J. Carmichael, S.J. Carvalho, A.A. Dominguez, T. Faith, J. Garcia, A.D. Gould, E.E. Jacobs, K. Kochan, C.M. Rubert Pérez, S.M. Rocus, G.B. Kharas. Int. J. Chem. Stud. 3 (4) 17-21 (2019).

[23] Novel Copolymers of Styrene. 4. Halogen Ring-Substituted Butyl 2-Cyano-3Phenyl-2-Propenoates. G.B. Kharas, Y. Gao, E.E. Merriman, K.N. Parker, H.T. Patel, M.B. Raza, J. Sekhon, S.M. Stuckey, M. Styrczula, M.C. Ulloa, and W.F. Vital. J. Macromol. Sci. A52 (11) 887-891 (2015).

[24] Synthesis and styrene copolymerization of novel halogen ring-substituted isopropyl 2cyano-3-phenyl-2-propenoates. Cimino, Alessandra; Kharas, Gregory B. Abstracts of Papers, 259th ACS National Meeting \& Exposition, Philadelphia, PA, United States, March 22-26, (2020), POLY-0387. https://doi.org/10.1021/scimeetings.0c05713

[25] SciFinder; Chemical Abstracts Service: Columbus, OH; https://scifinder.cas.org (accessed December 11, 2020).

[26] Smith, M. B.; March, J. Addition to Carbon-Hetero Multiple Bonds, In March's Advanced Organic Chemistry, J. Wiley \& Sons: New York, Ch.16, 1225, 2001. 